\title{
Neurophysiology in Spinal Cord Injury
}

\author{
Milan R. Dimitrijevic, M.D. \\ Department of Rehabilitation, Baylor College of Medicine, Houston, Texas 77030, \\ U.S.A.
}

It has long been recognised that spinal cord injury leads to paralysis. Until recent times the inevitable consequence was early death. With the improved management techniques which have been developed in post-war years and which have been chronicled in this Journal, the life expectancy of spinal cord injury victims has improved progressively and is now probably near normal.

As a consequence of these changes, our awareness of the clinical syndromes of chronic spinal cord injury has increased. In the meantime, the discipline of neurophysiology has become well established and extensive studies of spinal cord lesion models have been described, clearly shaping our views on mechanisms operating in our patients. It was from such experimental work that the hypothesis that spasticity was the consequence of dendritic sprouting in uninjured primary sensory neurons and interneurons emerged (Liu and Chambers, 1980). In spite of evidence to the contrary, many practicing physicians continue to adhere to this model, perhaps because of the observations that severe spasticity syndromes could be present in patients with 'spinal cord transection' with a segmental generator of hyperactivity below the level of the lesion.

The experimental neurophysiological work of Lundberg and colleagues on the brain influence on segmental reflexes led to two important new concepts. The first was based on observations of the convergence of segmental and suprasegmental influences on a pre-motor spinal cord centre located within the segmental spinal interneurons. This centre has the putative function of processing information prior to Sherrington's 'final common path'. The second indicated that certain segmental reflex activity was only present in the presence of brain bulbospinal influence (Lundberg, 1979).

With the development of clinical neurophysiology as the interface between experimental animal work and clinical practice in man, there arises an opportunity for furthering our understanding of mechanisms operating in our patients. Additional, systematic studies of segmental reflex in man by means of newly established methodologies-H-reflex, tendon jerks, cutaneomuscular, vibratory tonic and long latency reflexes, etc., have revealed at a clinical neurophysiological but clinically occult level residual suprasegmental influence on segmental 


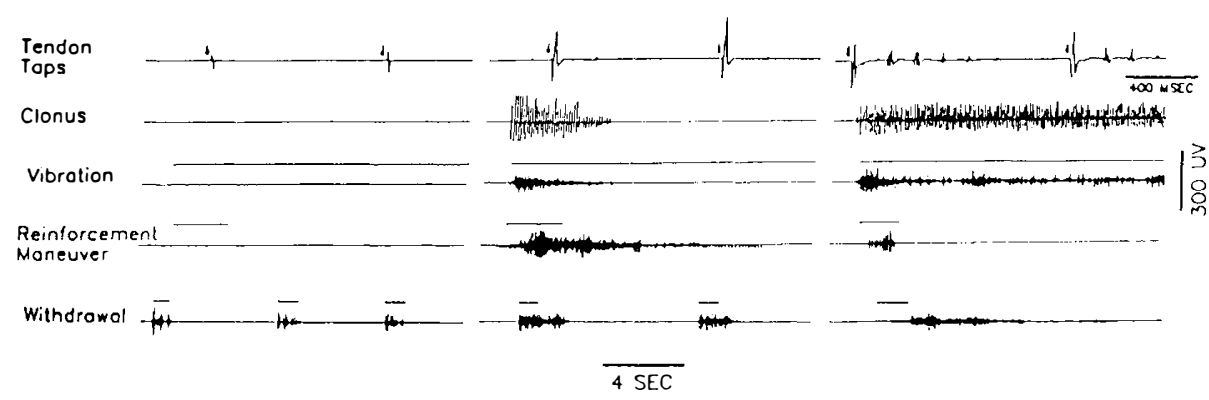

Figure 1 This figure is a summary of polyelectromyographic recordings in three patients: a patient with clinically and neurophysiologically complete lesion; a patient with clinically complete but neurophysiologically incomplete lesion-discomplete; and a patient with clinically and neurophysiologically incomplete lesion. Surface electrodes are placed over the quadriceps femoris in all three patients. Traces of electrical events from tendon taps, clonus, vibration, reinforcement maneuver and plantar withdrawl reflex are illustrated in the complete, discomplete and incomplete lesion.

reflexes in patients with the clinical picture of typical 'spinal cord transection' lesion (Fig. 1). Further evidence of suprasegmentally induced excitation and suppression in patients without motor activity has been obtained from such studies (Dimitrijevic and Faganel, 1985).

Thus, it is now clear that when spasticity arises in spinal cord injury patients there is residual brain influence with impaired suprasegmental influenceelementary, poorly controlled excitation and suppression in contrast to the highly integrated influence-on segmental reflex activity of the intact spinal cord. This, together with the direct evidence from microneurography that activity of large afferents of primary sensory neurons is not increased in patients with spasticity will in time force a change in the widely prevailing view of spasticity as being a consequence of a hyperactive gamma system with abnormally sensitive muscle spindle receptors (Brooks, 1986).

We need to move to the concept of spasticity as an abnormal central nervous system state reflecting abnormal brainstem influence with a degree of variability which reflects the extent of involvement of ascending and descending pathways. This view is similar to that of Magoun and Rhines (1947), who proposed that spasticity arises in the brainstem although here arising through residual and subclinical suprasegmental influence (Dimitrijevic, 1985).

Whatever satisfaction there may be in determining pathophysiological mechanisms, this remains a limited goal from the perspective of our patients. However, the determination of mechanisms can lead to the utilisation of therapeutic approaches with the treatment of spasticity with spinal cord stimulation as a case in point. Clinical neurophysiological techniques can also be used to demonstrate subclinical changes. Surface recorded lumbosacral evoked potentials have been used to demonstrate root potentials as well as pre- and post synaptic events from the posterior horns of the grey matter. Thus, it is possible to assess the functional state of the lumbosacral spinal cord even in patients with complete lesions at a more rostral level. Using this technique, the newly described syndrome of the 'double lesion' has emerged. Such studies have revealed in patients with sacral 

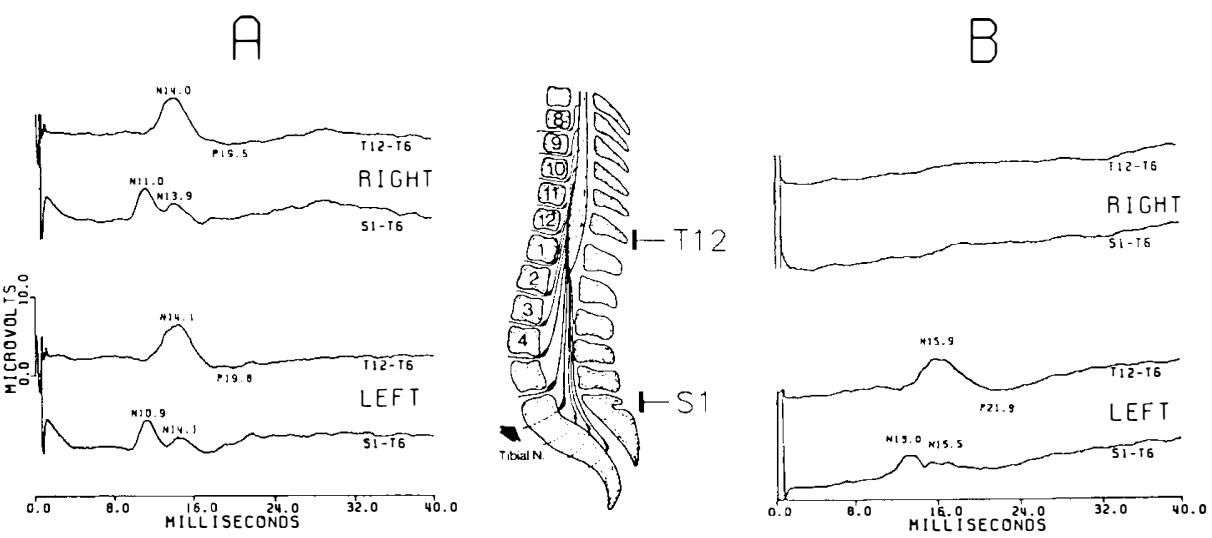

Figure 2 Lumbosacral somatosensory evoked potential of a neurologically healthy subject (A) and a patient with a cauda equina lesion (B). Surface electrodes are placed over T12 and S1 spinal processes: the tibial nerve is stimulated at the fossa poplitea. A recording between $\mathrm{T} 12$ and $\mathrm{T} 6$ represents a spinal cord event ( $\mathrm{S}$-wave) and between S1-T6 a nerve root event (R-wave). The stimulation of the right tibial nerve in the patient (upper B) elicits no electrospinogram whereas stimulation of the left tibial elicits a slightly altered but present electrospinogram. All events are averaged 128 times.

pain that the manifest, complete, rostral lesion is accompanied by a more caudal, occult lesion (Beric et al., in press) (Fig. 2).

Such findings lead us to re-examine our concepts of complete and incomplete spinal cord lesions. Between these two categories, clinical neurophysiological studies can clearly identify a new category of 'discomplete' spinal cord lesions. This discomplete category is represented by the patient who fulfills the clinical criteria of being complete with no volitional motor or sensory functions below

A

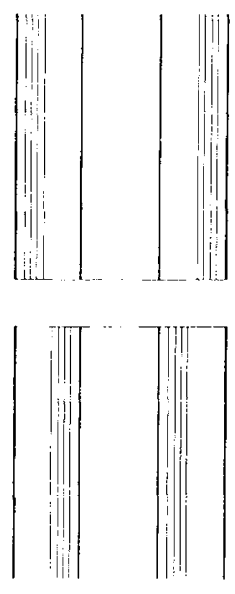

COMPLETE
B

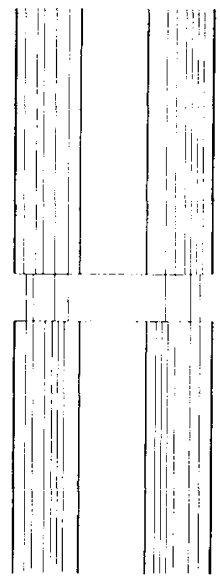

DISCOMPLETE
C

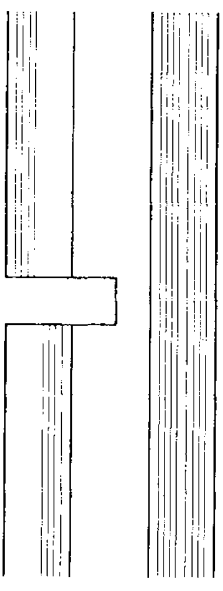

INCOMPLETE

Figure 3 In this figure there are three schematic drawings illustrating the main structural features of the spinal cord: (A) completely divided from the proximal part; (B) 'discomplete' lesion between the proximal and distal portions; (C) with incomplete division between the proximal and distal portions and with clinical findings for complete lesion but minimal structural connections. 
the level of the lesion yet has some residual motor function which can be demonstrated by the above laboratory techniques to indicate residual descending or ascending functions or control (Fig. 3).

In the near future we can expect that by using such techniques we will continue to advance our understanding of the mechanisms underlying the symptoms of our patients. By utilising this approach, we can go on to modify the clinical syndromes, leading to a further improvement in the quality of life of the survivors of spinal cord injury.

The majority of the work done at present relates to chronic spinal cord injury, but this approach is now being applied increasingly in the area of acute spinal cord injury. This will permit the development of protocols for neurophysiologically and actively supported recovery and guiding of neurobiological processes so that, hopefully, it will be possible to maximise the functional outcome for any given degree of injury.

\section{References}

Beric A, Dimitrijevic MR, Light KJ 1986 A clinical syndrome of rostral and caudal spinal injury: neurologic, neurophysiologic and urodynamic evidence for occult sacral lesion. Fournal of Neurology, Neurosurgery and Psychiatry. In press

BRooks VB 1986 The neural basis of motor control: Muscle tone. Oxford University Press, pp. 151-159

Dimitrijevic MR, Faganel J 1985 Motor control in the spinal cord. In: Eccles J, Dimitrijevic MR (eds) Recent Achievements in Restorative Neurology 1: Upper Motor Neuron Functions and Dysfunctions. S. Karger, Basel pp. 150-162

Dimitrijevic MR 1985 Spasticity. In: Swash M, Kennard C (eds) Scientific Basis of Clinical Neurology. Churchill Livingstone, pp. 108-115

LiU CN, CHAmbers WW 1980 Collateral sprouting in the spinal cord. In: Windel W (ed) The Spinal Cord and its Reaction to Traumatic Injury. Marcel Dekker, New York, pp. 219-236

LUNDBERg A 1979 Multisensory control of spinal reflex pathways. In: GRANit R, Pompeiano G (eds) Reflex control of posture and movement. Progress in Brain Research, Vol. 50.

Elsevier/North Holland Biomedical Press, Amsterdam, pp. 11-28

Magoun HW, Rhones R 1947 Spasticity. The stretch-reflex and extrapyramidal systems. Charles C Thomas, Springfield 